\title{
"It's Viral!" - A Study of the Behaviors, Practices, and Motivations of TikTok Users and Social Activism
}

\author{
Daniel Le Compte \\ daniellecompte@cmu.edu \\ Carnegie Mellon University \\ Pittsburgh, Pennsylvania, USA
}

\author{
Daniel Klug \\ dklug@andrew.cmu.edu \\ Carnegie Mellon University \\ Pittsburgh, Pennsylvania, USA
}

\begin{abstract}
Social media platforms such as Facebook and Twitter have long been used for social activism purpose, and lately the short-video app TikTok saw increased popularity for social activism content. We conducted 9 qualitative interviews with TikTok users who recently posted social activism content to better understand user motivations and creation practices when making TikTok videos related to social activism causes. We present an initial look into why TikTok is used for social activism, and what processes users use to carry out this work. Our interview analysis revealed the following five main themes: (1) motivation and inspiration for posting social activist content comes from a wide range of personal sources, (2) video creation practices largely match TikTok standards, (3) social activism has communities on TikTok that provide encouragement and discussion, (4) interaction with other activists and viewers is crucial for social activism content creation and (5) users choose TikTok for its ability to spread messages more simply and with wider range. These findings provide insight into the unique factors that TikTok brings for social activism and corroborates previous findings in understanding how social activists may use social media for their purposes.
\end{abstract}

\section{ACM Reference Format:}

Daniel Le Compte and Daniel Klug. 2021. "It's Viral!" - A Study of the Behaviors, Practices, and Motivations of TikTok Users and Social Activism. In Companion Publication of the 2021 Conference on Computer Supported Cooperative Work and Social Computing (CSCW'21 Companion), October 23-27, 2021, Virtual Event, USA. ACM, New York, NY, USA, 4 pages. https: //doi.org/10.1145/3462204.3481741

\section{LITERATURE REVIEW}

Social media platforms serve as "networked publics" [3], where there is a blend of technology, people, and practice, forming a unique space to allow people to gather for social, cultural, and civic purposes, that have special affordances that other publics do not offer. Specifically, a unique purpose of networked publics is social activism, seen on many social media platforms such as Facebook, Twitter, and now TikTok, for example, through the the rise of "Slacktivism" and information-distribution specific activism [16]. TikTok in particular has emerged as a unique social media platform that centers on user-generated content as its primary entertainment,

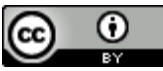

This work is licensed under a Creative Commons Attribution International 4.0 License.

CSCW'21 Companion, October 23-27, 2021, Virtual Event, USA

(c) 2021 Copyright held by the owner/author(s).

ACM ISBN 978-1-4503-8479-7/21/10.

https://doi.org/10.1145/3462204.3481741 discussion piece, and form of interaction [8, 17]. Interaction and discussion on TikTok largely occurs through three main features that, in some ways, resemble forums or threads on other platforms: 'duet' ${ }^{1}$, 'stitch' 2 , and 'comment in video' ${ }^{3}$. These characteristic forms of interaction are based on posted video content, therefore they are crucial for social activism purposes, such as education on new topics, prompting of collective action, and discourse on divisive issues.

\subsection{Social Activism on Social Media}

Activism in general has always relied on media in some form to get the message out and to encourage action. In recent years, social activism in particular has moved largely online to social media platforms as mainstream media formats have not suited activists' efforts or properly represented their views well $[6,19]$.

The specific dynamics and affordances for users to create and post social activist content and engage with an audience largely depends on the social media platform being used. For example, in a 'Reddit-like forum', one platform served as a central hub for communication and organizing for movements like the Anti-Extradition Bill Movement in Hong Kong [10]. Twitter, on the other hand, has been examined in greater detail by looking at how email chains and Twitter were combined and utilized to put together a cultural and literacy festival using decentralized networking [5], or how Twitter is being used for conversation and dialogue on political topics [2]. Further research focuses, for example, on immigrant activism [7], or on neighborhood community engagement via Facebook [12]. These examples showcase some of the many usages of social media for social activism, ranging from educational purposes, opinionated discussion and discourse, action calling and organizing movements, to humor, entertainment, and place-finding, and how these usages may vary dependent on the affordances of the platform.

In short, it is clear that social media platforms are utilized for a numerous set of purposes in social activism, and that these purposes are fulfilled on several different platforms, such as Twitter, Facebook, email networks, blogs and more. What is not fully understood, as of yet, is how TikTok plays a role in this system as a newer social media platform with unique characteristics based on video content and entertainment.

\subsection{Social Activism on TikTok}

TikTok recently became one of the most-downloaded social media platforms, and the academic community only started to study how TikTok is used to fulfill various social activist purposes [8]. Initial

\footnotetext{
${ }_{1}^{1}$ for example: https://vm.tiktok.com/ZMeGguGdt/ [August 3rd, 2021].

${ }^{2}$ for example: https://vm.tiktok.com/ZMeGgXnN4/ [August 3rd, 2021].

${ }^{3}$ for example: https://vm.tiktok.com/ZMdwE2LUw/ [August 6th, 2021].
} 
studies explore and evaluate aspects of action and organization, such as in the case of \#TulsaFlop with activists working against the Trump2020 campaign [1], or analyze how TikTok might be used by various groups for discussion, venting, and finding community [18] One poignant example of utilizing TikTok for social movements and awareness was a TikTok creator using her makeup videos as a platform to talk about the treatment of Uighurs by China in late 2019 [9]. While these studies show the initial usage of TikTok videos for various social activist purposes, they do not include a look into the motivations behind this type of content, how this content comes to fruition, or why some users are using these forms of user-generated content platforms for social activism over others. Based on this, our research was guided by the following questions: (1) Why and how do social activists in general use TikTok for social activism? (2) Do they use typical practices that are found elsewhere on TikTok, including popular or trending features? (3) Why do they use TikTok over other social media platforms? (4) What role does social activism on TikTok play in the greater work of social activism they do?

\section{METHOD: DATA COLLECTION AND ANALYSIS}

We conducted qualitative semi-structured interviews with nine TikTok users between March and April 2021. Participants had to have posted at least one video in the last month with some form of a social activist message. Participants were found by searching for popular activism hashtags on TikTok, such as \#socialactivism, \#stopasianhate, \#blm, or \#environmentalism, to identify content that could be classified as social activist content. Participants were then contacted to participate after they were identified as creators of this type of content. As of May 2021, participants were 18 to 30 years old, had 30 to $250 \mathrm{k}$ followers, posted 7 to $100+$ TikToks, and had a total of 185 to $8.6 \mathrm{~m}$ likes on their TikTok videos. Interview questions were broken up into four sections: About the participants' background as social activists, how they utilized TikTok, why they used TikTok for social activism, and how social activism on TikTok fits into their overarching process of activism.

The analysis of interviews followed an open coding process in which initial codes were generated based on a generative, iterative, build-up approach to coding, similar to other studies and methods found in HCI and CSCW research $[4,11,13]$. The first author analyzed the first interview transcript and created an initial set of codes that was edited with and agreed upon with the second author. This initial set of codes was used to code the remaining interviews, with an iterative approach to improve the clarity of the codes and emerging groups of codes. This process led to the creation of 149 codes that were structured into nine categories: audience engagement, content of videos, intended audience, motivation for using TikTok, overall usage of TikTok, tangible creation practices, TikTok specific features, trending features and items, and why TikTok was chosen over other platforms. These nine categories led to the identification of five themes described in the next section.

\section{RESULTS}

Our analysis of interview data resulted in identifying five main themes that describe how and why users post social activist content on TikTok: (1) motivation and inspiration for posting social activist content comes from a wide range of personal sources, (2) video creation practices largely match TikTok standards, (3) social activism has communities on TikTok that provide encouragement and discussion, (4) interaction with other activists and viewers is crucial for social activism content creation and (5) users choose TikTok for its ability to spread messages more simply and with wider range.

\subsection{Inspiration and Motivation}

Across all participants, inspiration and motivation for creating content came from multiple sources of a diverse range. For example, P02 described their area of work being an area they felt that they could contribute to the conversation: "Well, I can't talk that much about racism, (...) but I can talk about white saviorism because that's the industry that I live in" (P02). The industry of this participant's work motivated them to talk about issues they felt passionate about and were able to speak up about through TikTok videos. Two participants (P01, P04) described seeing a viewpoint or area of content on TikTok that they felt they needed to respond to as motivation for beginning to post social activist content to TikTok: "(...) and that truly began because a mutual friend of mine started sharing QAnon [content] all over his Instagram and he was great gaining a lot of traction and there was a lot of, um, anti mask protests popping up. And I was just like, I, I can't argue, I can't be bothered arguing in the comment section" (P04). Personal connection to a divisive topic is relevant and an important motivator for this participant. In many senses, the motivation for posting is deeply personal to participants, and is widely diverse and stems from many sources, whether careerrelated, familial, or other. This theme does not appear to vary by followership, frequency of posting, number of posted videos, or type of content posted.

\subsection{Creation Practices}

The creation practices of interviewees follow TikTok standards and are wide ranging and diverse, from improvisational and 'off the top-of-my-head' filming and creation, to more extensive practices that require research, preparation, and practice. One participant, in particular, exemplifies the casual, more improvisational, user persona: 'Um, you pretty much just kind of it's, it's this idea, this concept that I have in my head and it's pretty much all improv I speak" (P01). This exemplifies ideas of "Slacktivism" by Harlow and Guo [7], where activism is gaining popularity in simple, short, and low-effort methods to get a message out and to encourage participation. Additionally, this form of video creation follows a low-budget and minimal resources format: "(...) and then the actual filming, I mean, it's a quite low budget. I mean, it's just kinda like my phone, um, either natural lighting or with a ring light that I've got" (P05).

In addition to simple creation practices, almost all participants used some form of common editing techniques that are found on the TikTok platform, such as blending other content from outside of direct filming, duet-ing and stitching features, as well as using 
captions, images, and green-screen effects to create their videos. Other participants (P04, P05, P06), in addition, follow popular TikTok trends to get their messages across, such as popular filming styles, trending audio, as well as trending hashtags.

In contrast, a few participants (P01, P03, P04) used lengthy or involved processes used to create their messages and content. Of note, these three participants had medium to large followings $(26 \mathrm{k}, 250 \mathrm{k}$, $19 \mathrm{k}$ followers, respectively) and have posted frequently or for a long time. One participant (P03) in particular used a near "academic" process to research, study, and collect information and evidence about a topic, creating a "Master Document" that noted references and various points of opinion and evidence, before creating a script for a multi-part series of videos, one of which had 15 parts. This action seems to be not the norm, but not out of the scope of what participants would call necessary or useful for their type of content for activism.

\subsection{Social Activism Communities on TikTok}

In discussing why and how they started posting social activist content to TikTok, participants mentioned the notion of joining the social activist community on TikTok. One participant (P04) noted that the amount of content represented an opportunity to join, "Uh, but I saw so much social activism on there. I knew that there was an opportunity to do so there". Seeing connected content and other participants points this participant to an area where they can feel they can contribute and engage with others. Whereas, another participant noticed that there was a method and pattern of meeting other social activists on TikTok, "And they've [TikTok] really been pushing the social activism on my page, which is really cool. Cause I'm able to meet all these other social activists out there who inspire me to make my material" (P01). These comments point towards a connection between those who share similar values as an entrance point to the discussion as well as connections beyond the content and TikTok. This, in tandem with the next section of results, form the core motivations and usage of TikTok by participants interacting with and posting social activist content as a form of dialogue.

\subsection{Engagement and Interaction with Viewers is Crucial}

Across all participants was a desire to utilize many methods of interacting and engaging in discussion, discourse, and commentary on social activists topics and issues. For some participants, this became a core source of content and interaction with their audience members, such as one participant (P06) using suggestions from comments to create a "Abortion policy rating" system and video series for different countries. In their words of describing the reaction to this idea of interaction, “(...) getting people to comment and having this sort of format where you it's very, it's like interactive and it's fun because it's like, 'Oh my gosh, she's gonna give, she's gonna rate a legislation. That's so weird'” (P06). In this, they would take suggestions from their viewers to spark conversation and continue discussion on an important topic.

For other participants (P07, P08), the purpose of engagement and interaction with others on TikTok was largely a form of participating in social activism itself. One participant described their interactions as advocating for a particular belief or viewpoint: "And
I like to, um, advocate rather than discriminate. So if there's somebody who has a very rude comment, rather than backlashing them and calling them out and TikTok, I just explained to them like my side" (P01).

This format of engagement and interaction over a central topic, such as the Black Lives Matter movement, occurs in a different way than on other social media platforms. On TikTok, participants in conversation may create videos through stitches, duets, using comments in videos, or new videos entirely, to participate and engage. This is prevalent across all participants. One such example was the audio "I NEED YOU TO" by Tobe Nwigwe, which trended in mid-2020. Users created clickbait videos that started with the sound and showing an apparent trend but then suddenly switched to lip syncing the lyrics "Arrest the killers of Breonna Taylor" [14, 15]. This provides a unique avenue for discourse and fulfilling activists needs simultaneously, such as education and prompting action.

\subsection{Users Choose TikTok Due to its Ability to Spread Messages Widely}

When asking about the reason why participants use TikTok over other platforms for their social activist messages, one theme primarily emerged: TikTok as a platform allows for their content and messages to reach a much wider audience than what is possible with other platforms. Some participants noted that the usage of TikTok helps get their message out beyond their own 'circle': "So I was able to focus what my following was for rather than, um, Facebook, where it's like just friends of friends or family people you meet in real life" (P04). A main limiting factor of other platforms, that participants noted, was the necessity for audience members to connect or follow a creator before they would be able to see the content, unless in the unlikely event that the content was 'promoted' through ads, or went viral: "Well, I feel like TikTok, at least when I started using it, I felt like it was a lot easier for-for my videos to get sent to random people. Which is true, like on Instagram, unless I pay for it” (P05). In general, this feature and motivation for using TikTok was truly beneficial, as it also helped in the exchange of information and content between social activists and audience members alike: “(..) cause it really helps other people get their material to me. And it helps me get my material to other people" (P01). The motivation and reasoning for social activists to use TikTok connects to what may be found when we examine the early reasons for social activists to utilize social media in the first place. As noted by Watkins, to enact change, social activists desire to get the message across that fits their needs to the people that need to see it the most and that will be swayed into action or agreement the most [19]. For the participants in this study, TikTok fulfilled this role more than any other platform because interaction and engagement on TikTok is based on content and video feeds rather than on profiles and followers.

\section{CONCLUSION AND FUTURE WORK}

Overall, the findings of this study support previous research in attempting to understand how social activists use social media to carry out their efforts for social activism, such as education, discourse, and encouraging action. What this study brings new to the literature is a perspective on how and why users turn to TikTok over other social media platforms to create and share social activism 
content. The findings of motivation through engagement and interaction for people who post social activist content has implications for designing social media platforms; to better accommodate connection and communication, platforms may need to further account for varying preferences for this engagement and the aspects of motivation that prompt usage and interaction. Future work includes conducting additional interviews to further assess the differences in content, actions, and motivations for users posting social activist content between TikTok and other social media platforms. This study provides a unique perspective on social activism on social media, in particular how engagement and motivations to participate in the conversation is prevalent for the participants.

\section{REFERENCES}

[1] Jack Bandy and Nicholas Diakopoulos. 2020. \#TulsaFlop: A Case Study of Algorithmically-Influenced Collective Action on TikTok. FAccTRec (Dec. 2020), 1-7. http://arxiv.org/abs/2012.07716 arXiv: 2012.07716.

[2] Pablo Barberá, John T. Jost, Jonathan Nagler, Joshua A. Tucker, and Richard Bonneau. 2015. Tweeting From Left to Right: Is Online Political Communication More Than an Echo Chamber? Psychological Science 26, 10 (Oct. 2015), 1531-1542. https://doi.org/10.1177/0956797615594620 Publisher: SAGE Publications Inc.

[3] Danah boyd. 2010. Social Network Sites as Networked Publics: Affordances, Dynamics, and Implications. In A Networked Self: Identity, Community, and Culture on Social Network Sites (0 ed.), Zizi Papacharissi (Ed.). Routledge, England, UK, 47-66. https://doi.org/10.4324/9780203876527-8

[4] Kathy Charmaz. 1996. The Search for Meanings - Grounded Theory. In Rethinking Methods in Psychology. SAGE Publications Ltd, London, UK, 27-49.

[5] Judeth Oden Choi, James Herbsleb, and Jodi Forlizzi. 2019. Trust-Building Across Networks Through Festival Organizing. In Proceedings of the 9th International Conference on Communities \& Technologies - Transforming Communities. ACM, Vienna Austria, 300-305. https://doi.org/10.1145/3328320.3328403

[6] William A. Gameson and Gadi Wolfsfeld. 1993. Movements and Media as Interacting Systems. The Annals of the American Academy of Political \& Social Science 528 (1993), 114-125. https://doi.org/10.1177/0002716293528001009

[7] Summer Harlow and Lei Guo. 2014. Will the Revolution be Tweeted or Facebooked? Using Digital Communication Tools in Immigrant Activism. Fournal of Computer-Mediated Communication 19, 3 (April 2014), 463-478. https: //doi.org/10.1111/jcc4.12062

[8] D. Bondy Valdovinos Kaye, Xu Chen, and Jing Zeng. 2020. The co-evolution of two Chinese mobile short video apps: Parallel platformization of Douyin and TikTok. Mobile Media \& Communication 9, 2 (Aug. 2020), 229-253. https: //doi.org/10.1177/2050157920952120

[9] Lily Kuo. 2019. TikTok 'makeup tutorial' goes viral with call to action on China's treatment of Uighurs. http://www.theguardian.com/technology/2019/nov/ 27/tiktok-makeup-tutorial-conceals-call-to-action-on-chinas-treatment-ofuighurs Section: Technology.

[10] Francis L. F. Lee, Hai Liang, Edmund W. Cheng, Gary K. Y. Tang, and Samson Yuen 2021. Affordances, movement dynamics, and a centralized digital communication platform in a networked movement. Information, Communication \& Society (Feb. 2021), 1-18. https://doi.org/10.1080/1369118X.2021.1877772

[11] Nora Mcdonald, Sarita Schoenebeck, and Andrea Forte. 2019. Reliability and Inter-rater Reliability in Qualitative Research: Norms and Guidelines for CSCW and HCI Practice. Proc. ACM Hum.-Comput. Interact. 39, 39 (2019), 23. https: //doi.org/10.1145/3359174

[12] Gaia Mosconi, Matthias Korn, Christian Reuter, Peter Tolmie, Maurizio Teli, and Volkmar Pipek. 2017. From Facebook to the Neighbourhood: Infrastructuring of Hybrid Community Engagement. Computer Supported Cooperative Work (CSCW) 26, 4 (Dec. 2017), 959-1003. https://doi.org/10.1007/s10606-017-9291-z

[13] Joanne Neale. 2016. Iterative categorization (IC): a systematic technique for analysing qualitative data. Addiction 111, 6 (2016), 1096-1106. https://doi.org/10.1111/add.13314 _eprint: https://onlinelibrary.wiley.com/doi/pdf/10.1111/add.13314.

[14] Tobe Nwigwe. 2021. I NEED YOU TO created by Tobe Nwigwe | Popular songs on TikTok. https://www.tiktok.com/music/I-NEED-YOU-TO-6847297197650840326

[15] Kalhan Rosenblatt. 2020. Artist Tobe Nwigwe hopes viral song will reinvigorate calls for justice for Breonna Taylor. https://www.nbcnews.com/popculture/music/rapper-tobe-nwigwe-hopes-viral-song-will-reinvigorate-callsjustice-n1233879

[16] Dana Rotman, Sarah Vieweg, Sarita Yardi, Ed Chi, Jenny Preece, Ben Shneiderman, Peter Pirolli, and Tom Glaisyer. 2011. From slacktivism to activism: participatory culture in the age of social media. In Proceedings of the 2011 annual conference extended abstracts on Human factors in computing systems - CHI EA '11. ACM
Press, Vancouver, BC, Canada, 819. https://doi.org/10.1145/1979742.1979543

[17] Aliaksandra Shutsko. 2020. User-Generated Short Video Content in Social Media. A Case Study of TikTok. In Social Computing and Social Media. Participation, User Experience, Consumer Experience, and Applications of Social Computing, Gabriele Meiselwitz (Ed.). Vol. 12195. Springer International Publishing, Cham, 108-125. https://doi.org/10.1007/978-3-030-49576-3_8 Series Title: Lecture Notes in Computer Science.

[18] Sujatha Subramanian. 2021. Bahujan girls' anti-caste activism on TikTok. Feminist Media Studies 21, 1 (Jan. 2021), 154-156. https://doi.org/10.1080/14680777.2021. 1864875

[19] S. Craig Watkins. 2001. Framing Protest: News Media Frames of the Million Man March. Critical Studies in Media Communication 18, 1 (March 2001), 83. https://doi.org/10.1080/15295030109367125 Publisher: Taylor \& Francis Ltd. 